\title{
Obere und untere Schranken vollergodischer dynamischer Systeme mit quasidiskretem Spektrum
}

\author{
K. HäSLER
}

In jeder Klasse dynamischer Systeme induziert der Faktorbegriff eine Ordnungsstruktur, bezüglich der gemeinsame Faktoren und gemeinsame Vielfache zweier dynamischer Systeme studiert werden können. Diese Fragestellungen werden im folgenden speziell für die Klasse $\mathscr{X}^{*}$ der vollergodischen dynamischen Systeme mit quasidiskretem Spektrum untersucht und insbesondere Bedingungen dafür angegeben, wann maximale untere bzw. minimale obere Schranken in $\mathscr{K}^{*}$ existieren.

В каждом классе динамических систем повятие фактора определяет порядок, относительно которого можно изучить совместные факторы и совместные кратные для любых дивамических спстем. В последующем вопрос о совместных факторах и совместных кратвых рассматривается, в частности, в классе $\mathscr{K}^{*}$ вполне әргодических динамических систем. с квавидискретным спектром и даются условия существования для максимальных нпжных и мннимальных верхних границ в $\mathscr{X}^{*}$.

For every class of dynamical systems the factor conception induces an order structure. With respect to this structure one can regard common factors and common multiples of any two dynamical systems. In the following this question is studied for the class $\mathscr{K}^{*}$ of totally ergodic dynamical systems with quasidiscrete spectrum and conditions of existence and of uniqueness are considered for maximal lower and minimal upper bounds for any two systems of $\mathscr{K}^{*}$.

\section{Einleitnng}

Ist $(X, \mathscr{S}, m)$ ein Maßraum (im folgenden stets Lebesguesch) und $T$ eine maßtreue Transformation in $(X, \mathscr{S}, m)$, so heißt das Quadrupel $D=(X, \mathscr{S}, m, T)$ ein dynamisches System. Für dynamische Systeme hat sich insbesondere das Problem der Isomorphieinvarianten als wichtige und zugleich in vielen Fällen schwierige Fragestellung erwiesen.

Vollständige Lösungen dieses Problems sind z. B. für die Klassen der ergodischen Systeme mit diskretem Spektrum (durch J. v. NeUmans und P. Harmos, [7]), der vollergodischen Systeme mit quasidiskretem Spektrum (durch L. M. Abramov, [1]) und der Bernoulli-Schemata (durch D. Ornstens, [9]) gefunden worden. Eine umfassende Beschreibung des Isomorphieproblems in der Klasse der $K$-Automorphismen [10] liegt dagegen nicht vor.

Neben Isomorphiebetrachtungen haben in den letzten Jahren auch Untersuchun. gen über Faktoren dynamischer Systeme verstärktes Interesse gefunden. Ihr Zusammenhang mit Isomorphieproblemen liegt auf der Hand: Ist von zwei dynamischen Systemen jedes Faktor des anderen, so sind sie (wenigstens) zueinander schwach isomorph [13]. Zum Beispiel wurde die Frage der Faktorerblichkeit in vorgegebenen Klassen $\mathscr{D}$ dynamischer Systeme für wichtige Fälle untersucht. (Eine Klasse $\mathscr{D}$. wird faktorerblich genannt, wenn aus $D \in \mathscr{D}$ stets $D^{\prime} \in \mathscr{D}$ für jeden Faktor $D^{\prime}$ von $D$ folgt.) So konnte für ergodische dynamische Systeme mit diskretem Spektrum [7], 
für vollergodische dynamische Systeme mit quasidiskretem Spektrum [5] und Bernoulli-Schemata [10] Faktorerblichkeit nachgewiesen werden, was aber gerade in den letzten beiden Klassen nicht trivial ist. Das zeigt, daß trotz gut handhabbarer Isomorphieinvarianten Untersuchungen zur Faktorbeziehung in einer Klasse dynamischer Systeme ein kompliziertes Problem darstellen können. Die Gaußschen Systeme liefern ein Beispiel für eine nichtfaktorerbliche Klasse [8].

Auch bei den von H. Furstenbero [4] definierten Begriffen extremer Nichtisomorphie dynamischer Systeme (Disjunktheit, Teilerfremdheit) spielen Faktoren dynamischer Systeme eine wichtige Rolle.

Interessant sind ebenfalls Resultate, die sich mit der Kennzeichnung besonderer Faktoren beschäftigen. Der Satz von J. SrvaI, da B in jedes dynamische System mit positiver Entropie ein Bernoulli-Schema derselben Entropie eingebettet werden kann [13], die Ergebnisse von M. PrNsker über die Existenz eines maximalen Faktors der Entropie 0 für jedes dynamische System [12] oder die Untersuchungen von W. Parry über maximale Faktoren mit einer Darstellung als unipotente affine Transformationen auf Mannigfaltigkeiten für vollergodische dynamische Systeme [11] sind Beispiele für wichtige Ergebnisse in dieser Hinsicht.

Angeregt durch solche Resultate werden in dieser Arbeit in der Klasse $\mathscr{K}^{*}$ der vollergodischen dynamischen Systeme mit quasidiskretem Spektrum vor allem zwei Probleme untersucht :

a) Existenz und (eventuelle) Eindeutigkeit maximaler gemeinsamer Faktoren für zwei Systeme $D_{1}, D_{2} \in \mathscr{K}^{*}$,

b) Existenz und (eventuelle) Eindeutigkeit minimaler Vielfachen für zwei Systeme $D_{1}, D_{2} \in \mathscr{K}^{*}$.

In der Klasse $\mathscr{K}^{*}$ sind die Lösungen für die oben formulierten Probleme aus der Kenntnis des Isomorphieinvariantensystems nicht unmittelbar angebbar. Sie lassen den Strukturreichtum der Klasse $\mathscr{K}^{*}$ von einem anderen Betrachtungspunkt aus deutlich werden, als ihn Isomorphieuntersuchungen eröffnen.

Für eine Anwendung dieser Ergebnisse auf Disjunktheit dynamischer Systeme sei auf [6] verwiesen.

\section{Vollergodische dynamische Systeme mit quasidiskretem Spektrum}

Es sei $D=(X, \mathscr{S}, m, T)$ ein dynamisches System. Im folgenden wird der Hilbertraum der auf $X$ definierten, quadratisch integrierbaren Funktionen durch $L^{2}(X)$ bezeichnet werden, durch $U_{D}$ der durch den Automorphismus $T$ auf $L^{2}(X)$ induzierte isometrische Operator: $U_{D}(f)=f \circ T\left(f \in L^{2}(X)\right)$.

Für jedes dynamische System $D$ läßt sich die multiplikative Gruppe $G \subset L^{2}(X)$ der Funktionen $f \in L^{\infty}(X)$ betrachten, die die Eigenschaft $|f|=1 \mathrm{~m}$-f.ü. besitzen. Unter Benutzung der Restriktion des Operators $U_{D}$ auf $G$ läßt sich auf folgende Weise ein Endomorphismus $R_{D}$ auf $G$ definieren:

$$
R_{D}(f)=U_{D}(f) \cdot \bar{f} \quad(f \in G) .
$$

Daraus folgt unmittelbar die Beziehung

$$
U_{D}(f)=R_{D}(f) \cdot f \quad(f \in G) .
$$

Die Funktion $R_{D}(f)$ wird Quasieigenwert der Quasieigenfunktion $f \in G$ genannt. Auf der Grundlage dieser Festsetzungen bestimmt man für alle $n \in \mathbf{N} \cup\{0\}$ durch

$$
G_{n}(D)=R_{D}^{-n}(\{z \in \mathbf{C}:|z|=1\})=\left\{f \in G: R_{D}^{n}(f) \in K=\{z \in \mathbf{C}:|z|=1\}\right\}
$$


die Menge der Quasieigenfunktionen n-ter Ordnung (von $U_{D}$ ) und durch

$$
H_{n}(D) \doteq R_{D} G_{n}(D)
$$

die Menge der Quasieigenwerte n-ter Ordnung (von $U_{D}$ ). Entsprechend wird

$$
G(D)=\bigcup_{n=0}^{\infty} G_{n}(D)
$$

als Menge der Quasieigenfunktionen von $U_{D}$ und

$$
H(D)=\bigcup_{n=0}^{\infty} H_{n}(D)
$$

als Menge der Quasieigenwerte von $U_{D}$ bezeichnet. ${ }^{1}$ )

Für ergodische dynamische Systeme haben die eingeführten Begriffe folgende Eigenschaften:

1. Die Mengen $Q(D), H(D), G_{n}(D)$ und $H_{n}(D)(n \in N \cup\{0\})$, sind multiplikative, abelsche Gruppen.

2. Die Gruppe $H(D)$ ist höchstens abzählbar.

3. Die Abbildung $R_{D}$ ist ein lokal-nilpotenter Endomorphismus auf $H(D)$.

4. Es gilt $\left.H_{n}(D) \subseteq H_{n+1}(D) \subseteq G_{n}(D) \subseteq G_{n+1}(D)(n \in \mathbf{N} \cup\{0\}) .^{2}\right)$

5. $G_{n}(D) \neq G_{n+1}(D)$ gilt genau dann, wenn $H_{n}(D) \neq H_{n+1}(D)$ richtig ist, und es inmpliziert $G_{0}(D) \neq G_{1}(D) \neq \cdots \neq G_{n}(D)$ und $H_{0}(D) \neq H_{1}(D) \neq \cdots \neq H_{n}(D)$.

6. Gehören die Funktionen $f_{1}, f_{2} \in G(D)$ zum gleichen Quasieigenwert, so. gilt $f_{1}=c \cdot f_{2}$, wobei $c$ ein Element der Gruppe $K$ ist.

Ist die maßtreue Transformation $T$ vollergodisch, sind also alle Abbildungen $T^{\text {'n }}$ $(n \in \mathbf{N})$, ergodisch, so gilt zusätzlich noch folgende Eigenschaft:

7. Quasieigenfunktionen zu verschiedenen Quasieigenwerten sind orthogonal. ${ }^{3}$ )

Ein dynamisches System $D=(X, \mathscr{S}, m, T)$ heißt vollergodisch und von quasidiskretem Spektrum, wenn $T$ ein vollergodischer Automorphismus auf $(X, \mathscr{S}, m)$ ist und der Hilbertraum $L^{2}(X)$ aufgespannt wird durch die Gruppe der Quasieigenfunktionen $G(D)$ von $U_{D}$.

Jedem dynamischen System $D$ aus der Klasse $\mathscr{X}^{*}$ der vollergodischen dynamischen Systeme mit quasidiskretem Spektrum kann das $\operatorname{Paar}\left\langle H(D), R_{D}\right\rangle$ zugeordnet werden, das durch die Gruppe $H(D)$ seiner Quasieigenwerte und den zugehörigen Endomorphismus $R_{D}$ bestimmt wird. Aus dem Vorangegangenen geht hervor, daß die Komponenten jedes solchen Paares bestimmte algebraische Eigenschaften aufweisen. Das gibt Anla B, diese Paare, die den Elementen der Klasse $\mathscr{K}^{*}$ zugeordnet werden können, algebraisch zu beschreiben.

Definition. Ist $A$ eine torsionsfreie, abelsche, abzählbare Gruppe und $S$ ein auf $A$ definierter, lokal-nilpotenter Endomorphismus mit der Eigenschaft $A_{1}=\{a \in A: S a=1\} \subset K$, so wird das $\operatorname{Paar}\langle A, S\rangle$ eine Endomorphismus-Oruppe genannt.

1) Die Einführung der Begriffe der Quasieigenfunktionen und *werte für dynamische Systeme entspricht dem Vorgehen in der Arbeit [3], S. 215/216.

2) Für die Relation des mengentheoretischen Enthaltenseins wird im folgenden stets das Zeichen "@" verwendet. Soll das die Gleichhoit ausschließende Enthaltensein bezeichnet werden, so wird das Symbol ", $\subset$ " benutzt.

3) Zum Beweis der genannten Eigenschaften vgl. z. B. [1] oder [7]. 
Definition. 1. Zwei Endomorphismus-Gruppen $\langle A ; S\rangle$ und $\left\langle A^{\prime}, S^{\prime}\right\rangle$ werden äquivalent genannt (im Zeichen: $\langle A, S\rangle \simeq\left\langle A^{\prime}, S^{\prime}\right\rangle$ ), wenn ein Isomorphismus $V$. von $A$ auf $A^{\prime}$ existiert, der folgende Eigenschaften besitzt:
1. $\nabla(a)=a \quad\left(a \in A_{1}\right)$,
2. $V S(a)=S^{\prime} V(a) \quad(a \in A)$.

2. Zwei Endomorphismus-Gruppen $\langle A, S\rangle$ und $\left\langle A^{\prime}, S^{\prime}\right\rangle$ stehen in der Relation „ฏ“ zueinander (in Zeichen: $\langle A, S\rangle \leqq\left\langle A^{\prime}, S^{\prime}\right\rangle$ bzw. $\langle A, S\rangle \leqq\left\langle A^{\prime}, S^{\prime}\right\rangle$ mit der vermittelnden Abbildung $W$, falls der vermittelnde Homomorphismus $W$ von $A$ in $A^{\prime}$ für die Betrachtung von Bedeutung ist), wenn ein injektiver Homomorphismus $W$ von $A$ in $A^{\prime}$ existiert, der folgende Eigenschaften besitzt:

$$
\begin{aligned}
& \text { 1. } W(a)=a \quad\left(a \in A_{1}\right), \\
& \text { 2. } \left.W S(a)=S^{\prime} W(a) \quad(a \in A) .^{4}\right)
\end{aligned}
$$

Die Relation ,„ฏ“ bestimmt für Endomorphismus-Gruppen, benutzt man die angegebene Åquivalenzrelation, eine Ordnung. L. M. ABRAMOv wies in seiner Arbeit [1] den eineindeutigen Zusammenhang zwischen den Elementen der Klasse $\mathscr{X}^{*}$ und den Äquivalenzklassen von Endomorphismus-Gruppen nach. Es kann nicht nur jedem dynamischen System aus $\mathscr{K}^{*}$ eine Endomorphismus-Gruppe, nämlich das Paar $\left\langle H(D), R_{D}\right\rangle$, zugeordnet werden, auch die umgekehrte Beziehung ist richtig. $\mathrm{Zu}$ jeder Endomorphismus-Gruppe $\langle A, S\rangle$ existiert ein System $D \in \mathscr{K}^{*}$ derart, da $B$ $\langle A, S\rangle \simeq\left\langle H(D), R_{D}\right\rangle$ gilt.

L. M. Abramov bewies, daß die Menge der Åquivalenzklassen der Endomorphismus-Gruppen ein vollständiges System von Isomorphieinvarianten für die Klasse $\mathscr{K}^{*}$. bildet. Zwei dynamische Systeme aus $\mathscr{K}^{*}$ sind genau dann isomorph, wenn ihre Endomorphismus-Gruppen äquivalent sind. ${ }^{5}$ )

\section{Faktoren dynamischer Systeme aus $\boldsymbol{K}^{*}$}

Der Begriff des Faktors eröffnet eine Möglichkeit, Beziehungen zwischen dynamischen Systemen zu erschließen und darzustellen.

Definition. Ein dynamisches System $D_{1}=\left(X_{1}, \mathscr{S}_{1}, m_{1}, T_{1}\right)$ wird Faktor des dynamischen Systems $D_{2}=\left(X_{2}, \mathscr{S}_{2}, m_{2}, T_{2}\right)$ genannt, wenn es eine multiplikative Isometrie $V: L^{2}\left(X_{1}\right) \rightarrow L^{2}\left(X_{2}\right)$ gibt, die der Bedingung $V U_{D_{1}}=U_{D_{1}} V$ genügt. (In Zeichen: $D_{1} \leqq D_{2}$ oder, falls die Abbildung $\nabla$ für die Untersuchung von Bedeutung ist, $D_{1} \leqq D_{2}$ mit der vermittelnden Abbildung $V$.)

Für die Betrachtung des Faktorbegriffes für dynamische Systeme aus $\mathscr{K}^{*}$ lieferten F. HAHN und W. PARRY [5] einen wichtigen Beitrag. Sie wiesen nach, daß die Klasse $\mathscr{K}^{*}$ faktorerblich ist.

") Ist für zwei Endomorphismus-Gruppen, die in der Relation , $\leqq$ "zueinander stehen, wesentlich, daB sie nicht auch äquivalent sind, so wird das Symbol "§" ersetzt durch das Zeichen "<".

3) Zwei dynamische Systeme $D_{1}, D_{2}$ sind bekanntlich genau dann isomorph (= konjugiert in der Terminologie P. Halmos'), wenn es einen multiplikativen, isometrischen Operator $V$ : $L^{2}\left(X_{1}\right) \rightarrow L^{2}\left(X_{2}\right)$ mit folgenden Eigenschaften gibt:

1. $V L^{2}\left(X_{1}\right)=L^{2}\left(X_{2}\right)$,

2. $V U_{D 1}(f)=U_{D 2} V(f), \quad f \in L^{2}\left(X_{1}\right)$. 
Satz (Hatr, Parry). Ist $D_{2}$ ein Element der Klasse $\mathscr{X}^{*}$ und $D_{1}$ ein dynamisches System mit der Eigenschaft $D_{1} \leqq D_{2}$, so gilt $D_{1} \in \mathscr{K}^{*}$.

Die Untersuchung der Faktoren eines dynamischen Systems aus $\mathscr{X}^{*}$ führt wegen der Faktorerblichkeit dieser Klasse nicht aus ibr heraus. Da jedem System aus $\mathscr{K}^{*}$ auf eineindeutige Weise (mod. Ãquivalenz) eine Endomorphismus-Gruppe zugeordnet werden kann, wird sich die Beziehung zwischen einem System aus $\mathscr{X}^{*}$ und jedem seiner Faktoren in einer bestimmten Beziehung der zugehörigen Endomorphismus-Gruppen widerspiegeln. Der nachfolgende Satz zeigt diesen Zusammenhang auf. (Dabei wird auf die in Paragraph 2 betrachtete Ordnungsrelation für Endomorphismus-Gruppen Bezug genommen.)

Satz. Für zwei dynamische Systeme $D_{1}, D_{2}$ aus $\mathscr{K}^{*}$ gilt $D_{1} \leqq D_{2}$ genau dann, wenn für ihre Endomorphismus-Gruppen die Beziehung $\left\langle H\left(D_{1}\right), R_{D_{2}}\right\rangle \leqq\left\langle H\left(D_{2}\right), R_{D_{1}}\right\rangle$ richtig ist.

Beweis. 1. Gilt $D_{1} \leqq D_{2}$, existiert also eine multiplikative Isometrie $V: L^{2}\left(X_{1}\right)$ $\rightarrow L^{2}\left(X_{2}\right)$, die die Bedingung $V U_{D_{1}}=U_{D_{1}} V$ erfüllt, so lassen sich leicht folgende Eigenschaften des Operators $V$ nachprüfen:

1. $\operatorname{restr}_{H\left(D_{1}\right)} V: H\left(D_{1}\right) \rightarrow H\left(D_{2}\right)$.

2. restr $_{H\left(D_{1}\right)} V$ ist eine multiplikative und injektive Abbildung.

3. Es gilt $V(g)=g,\left(g \in H_{1}\left(D_{1}\right)\right)$.

4. Es ist $V R_{D_{1}}(g)=R_{D_{2}} V(g),\left(g \in H\left(D_{1}\right)\right)$, richtig.

Diese Eigenschaften zeigen, da $\left\langle H\left(D_{1}\right), R_{D_{1}}\right\rangle \leqq\left\langle H\left(D_{2}\right), R_{D_{z}}\right\rangle$ mit der vermittelnden Abbildung $W=$ restr $H\left(D_{1}\right) V$ von $H\left(D_{1}\right)$ in $H\left(D_{2}\right)$ gilt.

2. Es wird die Beziehung $\left\langle H\left(D_{1}\right), R_{D_{1}}\right\rangle \leqq\left\langle H\left(D_{2}\right), R_{D_{3}}\right\rangle$ mit der vermittelnden Abbildung $V: H\left(D_{1}\right) \rightarrow H\left(D_{2}\right)$ vorausgesetzt. Jeder Endomorphismus-Gruppe $\langle H, R\rangle$ kann ein unitärer Ring mit aufgeprägtem Automorphismus zugeordnet werden. (Vgl. [1], S. 522-524.) Der Raum $\mathscr{H}(\boldsymbol{H})$ aller auf $\boldsymbol{H}$ definierten, komplexwertigen Funktionen $f$, die der Bedingung

$$
\sum_{c \in H}|f(c)|^{2}<\infty
$$

genügen, wird mit folgendem Skalarprodukt und folgender Multiplikationsoperation ausgestattet:

$$
\begin{aligned}
& (f, g)=\sum_{c \in H} f(c) \cdot \overline{g(c)}, \\
& (f \cdot g)(c)=\sum_{\substack{a, b \in H \\
a, b=c}} f(a) \cdot g(b),
\end{aligned}
$$

wobei die Produktfunktion $f \cdot g$ existiert (in $\mathscr{H}(H)$ ), wenn

$$
\sum_{c \in H}\left|\sum_{\substack{a, b \in H \\ a . b=c}} f(a) \cdot g(b)\right|^{2}<\infty
$$

gilt $(f, g \in \mathscr{H}(\boldsymbol{H}))$.

Mit diesen Festlegungen wird $\mathscr{H}(H)$ zum unitären Ring. Durch die Bedingung $U\left(1_{a}\right)=Z(a) \cdot 1_{a} \cdot I_{R(a)}$ wird für die Elemente der Orthonormalbasis $\left\{I_{a}\right\}$ von $\mathscr{H}(H)$. die definiert sind durch

$$
I_{a}(c)=\left\{\begin{array}{l}
1, \text { falls } a=c, \\
0, \text { sonst, }
\end{array} \quad(a, c \in H),\right.
$$


ein isometrischer Operator konstruiert. Dabei ist $Z$ ein (beliebiger) Isomorphisnus der Gruppe $H$ in die Kreisgruppe $K$, der die Eigenschaft $Z(a)=\iota$ für alle $a \in H_{1}$ besitzt. Die lineare Fortsetzung von $U$ auf den gesamten Ring liefert einen multiplikativen, unitären Operator von $\mathscr{H}(H)$ auf sich.

Es seien im folgenden $\left(\mathscr{H}\left(H\left(D_{i}\right)\right), U_{i}\right)(i=1,2)$, unitäre Ringe mit aufgeprägten Automorphismen, die auf die angegebene Weise den Endomorphismus-Gruppen $\left\langle H\left(D_{i}\right), R_{D_{1}}\right\rangle$ zugeordnet sind. Man betrachtet nun die Endomorphismus-Gruppe $\left\langle V H\left(D_{1}\right), R_{D_{3}}\right\rangle$ und den zugehörigen unitären Ring mit aufgeprägtem Automorphismus $\left(\mathscr{H}\left(V H\left(D_{1}\right)\right), U\right)$, wobei der Automorphismus $U$ durch die Bedingung

$$
U\left(1_{a}\right)=Z(a) \cdot 1_{a} \cdot 1_{R_{D_{i}}(a)}
$$

mit $Z(a)=Z_{1} V^{-1}(a)\left(a \in V H\left(D_{1}\right)\right)$ festgelegt sei.

Durch die Bestimmung $W\left(I_{a_{1}}\right)=1_{V_{a_{1}}}\left(a_{1} \in H\left(D_{1}\right)\right)$ und anschließende lineare Fortsetzung des Operators $W$ auf den ganzen Raum $\mathscr{H}\left(H\left(D_{1}\right)\right)$ wird eine multiplikative Isometrie von $\mathscr{H}\left(H\left(D_{1}\right)\right)$ auf $\mathscr{H}\left(V H\left(D_{1}\right)\right)$ definiert, für die die Eigenschaft $U W(f)=W U_{\mathbf{i}}(f)\left(f \in \mathscr{H}\left(H\left(D_{1}\right)\right)\right)$ gilt.

Sowohl dem. unitären Ring mit Automorphismus $\left(\mathscr{H}\left(V H\left(D_{1}\right)\right), U\right)$ als auç dem unitären Ring mit Automorphismus $\left(\mathscr{H}\left(V H\left(D_{1}\right)\right), U_{2}\right)$ entspricht gemäß der Konstruktion zu Beginn dieses Beweisteils die Endomorphismus-Gruppe $\left\langle V H\left(D_{1}\right), R_{D_{2}}\right\rangle$. Es kann gezeigt werden, daß für jedes dynamische System $D \in \mathscr{K}^{*}$, dessen Endomorphismus-Gruppe $\left\langle H(D), R_{D}\right\rangle$ isomorph zu $\left\langle V H\left(D_{1}\right), R_{D_{2}}\right\rangle$ ist, multiplikative Isometrien

$$
S_{1}: L^{2}(X) \rightarrow \mathscr{H}\left(V H\left(D_{1}\right)\right) \text { und } S_{2}: L^{2}(X) \rightarrow \mathscr{H}\left(V H\left(D_{1}\right)\right)
$$

mit den Eigenschaften

und

$$
\dot{S}_{i}\left(L^{2}(X)\right)=\mathscr{H}\left(V H\left(\dot{D_{1}}\right)\right) \quad(i=1,2)
$$

$$
S_{1} U_{D}=U S_{1}, \quad S_{2} \dot{U}_{D}=U_{2} S_{2}
$$

existieren. (Vgl. [1].)

Man betrachtet nun den Operator $\mathscr{W}=S_{2} S_{1}^{-1} W$, der den unitären Ring $\mathscr{H}\left(H\left(D_{1}\right)\right)$ ị :den unịtären Ring $\mathscr{H}\left(H\left(D_{2}\right)\right)$, abbildet. Man zeigt leicht, daß die Abbildung $\mathscr{W}$ eine multiplikative Isometrie ist, die der Bedingung $\mathscr{W} U_{1}=U_{2} \mathscr{\mathscr { V }}$ genügt. Außerdem gilt: Die Spektraltypen $\left(L^{2}\left(X_{i}\right), U_{D_{i}}\right)$ besitzen die Eigenschaft: es existieren multiplikative Isometrien $\mathscr{W}_{i}: L^{2}\left(X_{i}\right) \rightarrow \mathscr{H}\left(H\left(D_{i}\right)\right)(i=1,2)$, wobei $\mathscr{W}_{i}\left(L^{2}\left(X_{i}\right)\right)$ $=\mathscr{H}\left(H\left(D_{i}\right)\right)$ und $\mathscr{W}_{i} U_{D_{i}}=U_{i} \mathscr{W}_{i}(i=1,2)$ zutrifft. (Vgl. [1].)

Daher ist die Beziehung $D_{1} \leqq D_{2}$ mit der die Faktoreigenschaft vermittelnden Abbildung $S=\mathscr{W}_{2}^{-1} \mathscr{F}_{\mathscr{W}}$ richtig.

Bemerkung.

1. Der vorangegangene Satz ermöglicht eine einfache Beweisführung für den bekannten Sachverhalt, daß in der Klasse $\mathscr{K}^{*}$ die Begriffe der starken und der schwachen Isomorphie übereinstimmen. L. M. ABramov bewies, daß zwei dynamische Systeme aus $\mathscr{K}^{*}$ genau dann (stark) isomorph sind, wenn ihre EndomorphismusGruppen $\left\langle H\left(D_{1}\right), R_{D_{1}}\right\rangle$ und $\left\langle H\left(D_{2}\right), R_{D_{3}}\right\rangle$ äquivalent sind [1]. Nach der Definition von J. SrNar [13] werden zwei Systeme $D_{1}, D_{2}$ schwach isomorph genannt, wenn sowohl $D_{1} \leqq D_{2}$ als auch $D_{2} \leqq D_{1}$ gilt. Der vorangegangene Satz zeigt, da $B$ schwache Isomorphie für dynamische Systeme $D_{1}, D_{2}$ aus $\mathscr{K}^{*}$ gleichbedeutend ist mit dem gleichzeitigen Gelten der beiden Relationen

$$
\left\langle H\left(D_{1}\right), R_{D_{1}}\right\rangle \leqq\left\langle H\left(D_{2}\right), R_{D_{2}}\right\rangle \quad \text { und } \quad\left\langle H\left(D_{2}\right), R_{D_{2}}\right\rangle \leqq\left\langle H\left(D_{1}\right), R_{D_{1}}\right\rangle \text {. }
$$


Da die $\leqq$-Relation für Endomorphismus-Gruppen aber eine Ordnung ist; _entspricht das gerade der Äquivalenz der zwei Endomorphismus-Gruppen.

2. Der angegebene Satz ermöglicht einfache Folgerungen, die vor allem die praktische Untersuchung über das Verhältnis von zwei vorgegebenen dynamischen Systemen aus $\mathscr{K}^{*}$ erleichtern können:

So folgt für zwei Systeme $D_{1}, D_{2}$ aus $\mathscr{K}^{*}$ aus der Beziehung $D_{1} \leqq D_{2}$ stets $H_{1}\left(D_{1}\right) \cong H_{1}\left(D_{2}\right)$; die Umkehrung dieses Satzes ist natürlich i. a. falsch.

Bezeichnet man durch $n\left(D_{1}\right), n\left(D_{2}\right)$ die Halmos-Invarianten der Systeme $D_{1}, D_{2}^{\prime \prime}$ aus $\mathscr{K}^{*}$, dann ergibt sich mit obigem Satz unmittelbar: Die Beziehung $D_{1} \leqq D_{2}$ hat die Eigenschaft $n\left(D_{1}\right) \leqq n\left(D_{2}\right)$ zur Folge.

\section{Untere Schranken dynamischer Systeme aus $\mathscr{K}^{*}$}

Bei der Strukturuntersuchung der Klasse $\mathscr{K}^{*}$ mit Hilfe des Faktorbegriffes ist es naheliegend, nach der Beschaffenheit der gemeinsamen Faktoren zweier oder mehr dynamischer Systeme aus $\mathscr{K}^{*}$ zu fragen. Im folgenden wird nur die Memge der gemeinsamen Faktoren für beliebige zwei Systeme aus $\mathscr{K}^{*}$ ùtersucht. Die angeführten Resultate lassen sich.aber ohne Schwierigkeiten auf den Fall. einer beliebigen endlichen Zahl von Systemen aus $\mathscr{K}^{*}$ verallgemeinern. Die Betrachtung einer abzählbaren oder ïberabzählbaren Anzahl von Elementen aus $\mathscr{K}^{*}$ bezüglich der ihnen allen gemeinsamen Faktoren bedarf neuer Utberlegungen.

Definition. Sind $D_{1}, D_{2}$ Elemente aus $\mathscr{K}^{*}$, so wird ein dynamisches System $D_{0}$ untere Schranke von $D_{1}$ und $D_{2}$ genannt, wenn $D_{0} \leqq D_{i}(i=1,2)$ gilt. Ein dynamisches System $D_{0}$ wird maximale untere Schranke von $D_{1}$ und $D_{2}$ genannt, wenn gilt:

1. $D_{0}$ ist untere Schranke von $D_{1}$ und $D_{2}$.

2. Für jedes dynamische System $D$ mit der Eigenschaft $D_{0} \leqq D \leqq D_{i}(i=1 ; 2)$ ist $D_{0} \simeq D$ richtig.

Bezeichnung. Die Menge der unteren Schranken zweier Systeme $D_{1}$ und $D_{2}$ wird durch $\mathscr{U}\left(D_{1}, D_{2}\right)$ bezeichnet, die Menge der maxinialen unteren Schranken durch $\mathscr{U}_{*}\left(D_{1}, D_{2}\right)$.

Die Definitionen der unteren und der maximalen unteren Schranke können auf die angegebene Weise für Elemente aus beliebigen Klassen dynamischer Systemie getroffen werden. Die faktorerbliche Klasse $\mathscr{K}$ der ergodischen dynamischen Systeme mit diskretem Spektrum liefert eine einfache Möglichkeit dafür, sich die betrachteten Mengen $\mathscr{U}\left(D_{1}, D_{2}\right)$ und $\mathscr{U}_{*}\left(D_{1}, D_{2}\right)\left(D_{1}, D_{2} \in \mathscr{K}\right)$ am speziellen Beispiel zu veranschaulichen. Benutzt man das von Neumannsche vollständige System von -Isomorphieinvarianten für die Klasse $\mathscr{K}$, so lassen sich leicht folgende Zusammenhänge nachprüfen: Die Menge $\mathscr{T}\left(D_{1}, D_{2}\right)$ wird für beliebige zwèi Systeme $D_{1}, D_{2}$ aus $\mathscr{K}$ durch alle Elemente aus $\mathscr{K}$ gebildet, deren Eigenwertgruppen enthalten sind im Durchschnitt der. Eigenwertgruppen von $D_{1}$ und $D_{2}$. Als (mod. Isomorphie) eindeutig bestimmte maximale untere Schranke von $D_{1}$ und $D_{2}$ erweist sich dasjenige System aus $\mathscr{K}$, dessen Eigenwertgruppe glẹich dem Durchschnitt der Eigenwertgruppen von $D_{1}$ und $D_{2}$ ist.

Die Mengen $\mathscr{U}\left(D_{1}, D_{2}\right)$ und $\mathscr{U}_{*}\left(D_{1}, D_{2}\right)$ weisen für beliebige Systeme $D_{1}, D_{2}$ aus $\mathscr{K}^{*}$ entsprechende Eigenschaften auf. Mit Hilfe des Faktorkriteriums für $\mathscr{K}^{*}$ können die Elemente aus $\mathscr{U}\left(D_{1}, D_{2}\right)\left(D_{1}, D_{2} \in \mathscr{K}^{*}\right)$ durch Forderungen an die Beschaffenheit ihrer Endomorphismus-Gruppen beschrieben werden. Es läßt sich zeigen, daß die Menge $\mathscr{U}_{*}\left(D_{1}, D_{2}\right)$ für beliebige Systeme $D_{1}, D_{2}$ aus $\mathscr{K}^{*}$ (bis auf Isomorphie) genau ein Element enthält. Der Nachweis hierfür soll in zwei Schritten erfolgen. 
Satz. Gilt $D_{1}, D_{2} \in \mathscr{K}^{*}$ und $D \in \mathscr{U}\left(D_{1}, D_{2}\right)$, so existiert ein dynamisches System $D_{0} \in \mathscr{U}_{*}\left(D_{1}, D_{2}\right)$ mit der Eigenschaft $D \leqq D_{0}$.

Beweis. Es seien

$$
D \in \mathscr{U}\left(D_{1}, D_{2}\right) \quad \text { und } \quad \mathscr{U}=\left\{D^{\prime} \in \mathscr{U}\left(D_{1}, D_{2}\right): D \leqq D^{\prime}\right\}
$$

$\mathscr{U}$ ist nicht leer. Der Faktorbegriff für dynamische Systeme induziert für die Menge $\mathscr{U}$ eine Ordnung. Für Systeme $D^{\prime}, D^{\prime \prime} \in \mathscr{U}$ gelte $D^{\prime}<D^{\prime \prime}$ genau dann, wenn $D^{\prime} \leqq D^{\prime \prime}$ richtig ist. Mit dieser Ordnungsrelation versehen, ist $\mathscr{U}$ induktiv geordnet. Ist $\left(D_{.}^{\prime}\right)$ ( $\iota \in I=$ Indexmenge) eine beliebige Kette aus $\mathscr{U}$, dann gilt gemäß $\S 2$

$$
\left\langle H\left(D_{\imath}^{\prime}\right), R_{D^{\prime}}\right\rangle \leqq\left\langle H\left(D_{\imath+1}^{\prime}\right), R_{D_{\imath}+1}\right\rangle
$$

für alle $\iota \in I$. Der induktive Limes dieser Endomorphismus-Gruppen (bezüglich des Homomorphismus-Systems $\left(V_{l}\right)$ ) ist (mod. Aquivalenz) eine EndomorphismusGruppe, die die Eigenschaft besitzt, daß das durch sie bestimmte dynamische System aus $\mathscr{K}^{*}$ sämtliche Systeme $D_{\imath}^{\prime}(\iota \in I)$ zu Faktoren hat und gleichzeitig ein Element in $\mathscr{U}$ ist. $^{6}$ )

Die Anwendung des Zornschen Lemmas auf die geordnete Menge $\mathscr{l}$ erbringt den Beweis für die Existenz eines maximalen Elementes in $\mathscr{U}$, das wegen der Konstruktion von $\mathscr{U}$ und $\mathscr{U}_{*}\left(D_{1}, D_{2}\right)$ auch zu $\mathscr{U}_{*}\left(D_{1}, D_{2}\right)$ gehört.

Hilfssatz. Gilt $D_{1}, D_{2} \in \mathscr{X}^{*}$ und $D, D^{\prime} \in \mathscr{Q}_{*}\left(D_{1}, D_{2}\right)$, so ist für die Gruppen der Quasieigenwerte zweiter Ordnung $\mathrm{H}_{2}(D), \mathrm{H}_{2}\left(D^{\prime}\right)$ die folgende Beziehung richtig:

$$
\left\langle H_{2}(D), R_{D}\right) \simeq\left\langle H_{2}\left(D^{\prime}\right), R_{D^{\prime}}\right\rangle \text {. }
$$

Beweis. Es seien $D^{(2)}$ und $D^{\prime(2)}$ die zu den Endomorphismus-Gruppen $\left\langle H_{2}(D), R_{D}\right\rangle$ bzw. $\left\langle H_{2}\left(D^{\prime}\right), R_{D^{\prime}}\right\rangle$ gehörigen Systeme aus $\mathscr{K}^{*}$. O. B. d. A. kann angenommen werden, daß die vermittelnden Transformationen für die Faktorbeziehungen

$$
\left\langle H_{2}(D), R_{D}\right\rangle \leqq\left\langle H\left(D_{1}\right), R_{D_{1}}\right\rangle \text { und }\left\langle\dot{H}_{2}\left(D^{\prime}\right), R_{D^{\prime}}\right\rangle \leqq\left\langle H\left(D_{1}\right), R_{D_{1}}\right\rangle
$$

die identischen Abbildungen sind. Für die Beziehung

$$
\left\langle H_{2}(D), R_{D}\right\rangle \leqq\left\langle H\left(D_{2}\right), R_{D_{2}}\right\rangle
$$

wird die vermittelnde Abbildung durch $W$, für

$$
\left\langle H_{2}\left(D^{\prime}\right), R_{D^{\prime}}\right\rangle \leqq\left\langle H\left(D_{2}\right), R_{D_{2}}\right\rangle
$$

durch $W^{\prime}$ bezeichnet.

1. Konstruktion eines Elementes $D_{0} \in \mathscr{U}\left(D^{(2)}, D^{\prime(2)}\right)$. Die Menge

$$
H=\left\{h \in H_{2}(D): \exists h^{\prime} \in H_{2}\left(D^{\prime}\right) \quad \text { mit }{ }^{\prime} R_{D}(h)=R_{D^{\prime}}\left(h^{\prime}\right)\right\}
$$

bildet offensichtlich eine torsionsfreie, abelsche Gruppe, deren Kern bezüglich $R_{D}$ eine Untergruppe der Kreisgruppe $K$ ist. Es gilt ker $R_{D} \cap H=H_{1}(D)$. Die Gruppe $H$ kann vermittels eines Isomorphismus $V$ in die Gruppe $H_{2}\left(D^{\prime}\right)$ abgebildet werden:

Für jedes $h \in H_{1}(D) \cap H=H_{1}(D)$ bestimmt man $V(h)=h$. Existiert ein Element $h \in H \backslash H_{1}(D)$, so erweitert man den Isomorphismus $V$ auf die Gruppe $\tilde{H}$, die erzeugt wird von $H_{1}(D)$, allen Vielfachen von $h$ und allen Wurzeln von $h$, die in $H$ liegen, auf folgende Weise: Besitzt $h$ keine Wurzel in $H$, so setzt man $V(h)=c \cdot h$, wobei $c \in H_{1}\left(D_{1}\right)$ so gewählt ist, $\operatorname{da} \beta V(h)=c \cdot h \in H\left(D^{\prime}\right)$ gilt. Hat $h$ eine Wurzel in $H$,

6) Zur Konstruktion des inversen Limes vergleiche man [2] (besonders Theorem 1) unter Beachtung der Faktorerblichkeit der Klasse $\mathscr{X}^{*}$. 
existiert also eine Zahl $n \in \mathbf{N} \backslash\{1\}$ mit $\sqrt[n]{h} \in H$, so bestimmt man $V(\sqrt[n]{h})=c^{\prime} \cdot \sqrt[n]{h}$ $\in H\left(D^{\prime}\right)$ mit geeignetem $c^{\prime} \in H_{1}\left(D_{1}\right)$. Gehören zwei Wurzeln von $h$ zu $H$, d. h. gibt es natürliche Zahlen $m$ und $n(m \neq n, m \neq 1, n \neq 1)$ mit $\sqrt[m]{h} \in H$ und $\sqrt[n]{h} \in H$, so ist auch $\sqrt[m \cdot n]{h^{k}} \in \boldsymbol{H}$ richtig, wobei $k$ der größte gemeinsame Teiler von $m$ und $n$ ist. Man definiert.

$$
V\left(\sqrt[m \cdot n]{h^{k}}\right)=c^{\prime \prime} \cdot \sqrt[m \cdot n]{h^{k}} \in H\left(D^{\prime}\right)
$$

mit geeignetem $c^{\prime \prime} \in H_{1}\left(D_{1}\right)$. Induktive Fortsetzung des Verfahrens führt wegen der Abzählbarkeit von $H$ nach höchstens abzählbar vielen Schritten auf eine Wurzel $\tilde{h}$ von $h$ in $H$ mit der Eigenschaft, daß alle anderen Wurzeln von $h$ in $H$ Vielfache von $\bar{h}$ sind. Man bestimmt $V(\tilde{h})=c \cdot \bar{h} \in H\left(D^{\prime}\right)$ mit geeignetem $c \in H_{1}\left(D_{1}\right)$. Die Abbildung $V$ kann auf $\tilde{H}$ multiplikativ fortgesetzt werden. Gilt $\{\tilde{\tilde{h}}\} \subseteq H \backslash \tilde{H}$, so wendet man die eben benutzte Konstruktion zur Erweiterung von $V$ auf die Gruppe $\widetilde{H}$ an, die erzeugt wird durch $\tilde{H}$, alle Vielfachen von $\tilde{h}$ und alle Wurzeln von $\tilde{h}$ in $H$. Nach höchstens abzählbar vielen Schritten endet auch dieses Vorgehen wegen der Abzählbarkeit von $H$. Aus der Bestimmung von $V$ folgt unmittelbar, da $B R_{D_{1}} V=V R_{D_{1}}$ gilt. Das dynamishe System $D_{0} \in \mathscr{K}^{*}$, das die Endomorphismus-Gruppe $\left\langle H\left(D_{0}\right), R_{D_{0}}\right\rangle \simeq\left\langle H, R_{D_{1}}\right\rangle$ besitzt, ist daher ein Element aus $\mathscr{T}\left(D^{(2)}, D^{\prime(2)}\right)$.

2. Es gilt $D_{0} \in \mathscr{U}_{*}\left(D^{(2)}, D^{\prime(2)}\right)$.

Für ein beliebiges System $\tilde{D} \in \mathscr{U}\left(D^{(2)}, D^{\prime(2)}\right)$ ist nänlich folgendes richtig:

$$
H_{1}(\tilde{D}) \subseteq H_{1}\left(D^{(2)}\right) \cap H_{1}\left(D^{\prime(2)}\right)=H_{1}\left(D_{0}\right) \text {. }
$$

Außerdem existieren für beliebiges Element $\tilde{h} \in \boldsymbol{H}(\tilde{D})$ nach Voraussetzung über $\tilde{D}$ Funktionen $h \in H_{2}(D)$ bzw. $h^{\prime} \in H_{2}\left(D^{\prime}\right)$ mit der Eigenschaft $Z(\tilde{h})=h$ und $Z^{\prime}(\tilde{h})=h^{\prime}$, wenn man $\tilde{D} \leqq D^{(2)}$ mit der vermittelnden Abbildung $Z$ und $\tilde{D} \leqq D^{\prime(2)}$ mit der vermittelnden Äbildung $Z^{\prime}$ setzt. Wegen

$$
R_{D}(h)=R_{D} Z(\tilde{h})=Z R_{\tilde{D}}(\tilde{h})=R_{\tilde{D}}(\bar{h})=Z^{\prime} R_{\tilde{D}}(\bar{h})=R_{D^{\prime}} Z^{\prime}(\tilde{h})=R_{D^{\prime}}\left(h^{\prime}\right)
$$

ist $h=Z(\bar{h}) \in H$ richtig. Es gilt daher

$$
\left\langle H(\tilde{D}), R_{\tilde{D}}\right\rangle \leqq\left\langle H, R_{D}\right\rangle \simeq\left\langle H\left(D_{0}\right), R_{D_{0}}\right\rangle
$$

mit der vermittelnden Abbildung $Z: H(\tilde{D}) \rightarrow H$.

Mithin ist das System $D_{0} \in \mathscr{K}^{*}$ eindeutig bestimmte maximale untere Schranke von $D^{(2)}$ und $D^{\prime(2)}$.

3. Wie in $\S 5$ angegeben, konstruiere man zu den Systemen $D^{(2)}$ und $D^{\prime(2)}$ unter Benutzung ihrer maximalen unteren Schranke $D_{0}$ ein dynamisches System $D^{*}$ aus $\mathcal{O}^{*}\left(D^{(2)}, D^{\prime(2)}\right)$. Es gelte also

$$
\begin{aligned}
& \left(L^{2}\left(X_{0}\right), U_{D_{0}}\right) \leqq\left(L^{2}\left(X^{(2)}\right), U_{D}\right) \text { mit der vermittelnden Abbildung } V, \\
& \left(L^{2}\left(X^{(2)}\right), U_{D}\right) \leqq\left(L^{2}\left(X^{*}\right), U_{D^{*}}\right) \text { mit der vermittelnden Abbildung } W, \\
& \left(L^{2}\left(X_{0}\right), U_{D_{0}}\right) \leqq\left(L^{2}\left(X^{\prime(2)}\right), U_{D^{\prime}}\right) \text { mit der vermittelnden Abbildung } V^{\prime}
\end{aligned}
$$

und

$$
\left(L^{2}\left(X^{\prime}(2)\right), U_{D^{\prime}}\right) \leqq\left(L^{2}\left(X^{*}\right), U_{D^{*}}\right) \text { mit der vermittelnden Abbildung } W^{\prime},
$$

wobei die Eigenschaft $W V\left(f_{0}\right)=W^{\prime} V^{\prime}\left(f_{0}\right)$ für alle Funktionen $f_{0} \in L^{2}\left(X_{0}\right)$ gilt.

Dabei ist $H_{1}\left(D^{*}\right)=H_{1}(D)=H_{1}\left(D^{\prime}\right)$ richtig. Gilt nämlich

d. h.

$$
R_{D^{*}}\left(h^{*}\right)=R_{D^{\bullet}}\left(W h \cdot W^{\prime} h^{\prime}\right)=1 \quad\left(W h \in W H_{2}(D), \quad W^{\prime} h^{\prime} \in W^{\prime} H_{2}\left(D^{\prime}\right)\right),
$$

$$
R_{D^{*}} W(h)=W R_{D}(h)=W^{\prime} R_{D^{\prime}}\left(h^{\prime-1}\right)=R_{D^{\bullet}} W^{\prime}\left(h^{\prime-1}\right) \in H_{1}\left(D_{0}\right),
$$


wie unmittelbar aus den Bedingungen für $W(h)$ und $W^{\prime}\left(h^{\prime}\right)$ folgt, ergibt sich $W(h) \in W H$. Wegen der Ergodizität von $D^{*}$ (vgl. $\S 5$, Hilfssatz 5), erhält man $W(h)=c \cdot W^{\prime}\left(h^{\prime-1}\right), c \in K$. Aus den Voraussetzungen über $D_{0}$ und die Abbildungen $V, W, V^{\prime}, W^{\prime}$ folgt sofort $c \in H_{1}\left(D_{0}\right)=H_{1}(D)=H_{1}\left(D^{\prime}\right)$. Daher gilt $h^{*}=c \in H_{1}\left(D_{0}\right)$.

Die Eigenschaft $H_{1}\left(D^{*}\right)=H_{1}\left(D_{0}\right)$ beweist, daß $D^{*}$ vollergodisch ist: Gemäß Hilfssatz 4 und 5 aus $\S 5$ ist $D^{*}$ ergodisch und besitzt quasidiskretes Spektrum. Aus der Torsionsfreiheit von $H_{1}\left(D^{*}\right)$ folgt nun sogar $D^{*} \in \mathscr{K}^{*}$.

4. $D^{*}$ ist untere Schranke von $D_{1}$ und $D_{2}$.

Man konstruiert dazu entsprechend wie in Beweisteil 1 eine untere Schranke von $D^{*}$ und $D_{i}$, indem man definiert

$$
\begin{aligned}
& H_{i}=\left\{h^{*} \in H_{2}\left(D^{*}\right)=H\left(D^{*}\right): \exists h_{i} \in H_{2}\left(D_{i}\right) \text { mit } R_{D^{*}}\left(h^{*}\right)=R_{D_{1}}\left(h_{i}\right)\right\} \\
& (i=1,2) .
\end{aligned}
$$

Aus der Konstruktion von $D^{*}$ folgt dann unmittelbar, daß $\left\langle H_{i}, R_{D_{i}}\right\rangle \simeq\left\langle H\left(D^{*}\right), R_{D^{*}}\right\rangle$ richtig ist. Das heißt, es gilt $D^{*} \leqq D_{i}(i=1,2)$. Das ist mit der Voraussetzung $D, D^{\prime} \in u_{*}\left(D_{1}, D_{2}\right)$, d. h. speziell $\bar{D}^{(2)}, D^{\prime(2)} \in \mathscr{U}_{*}\left(D_{1}^{(2)}, D_{2}{ }^{(2)}\right)$, nur dann vereinbar, wenn $D^{(2)} \simeq D^{*} \simeq D^{\prime(2)}$ richtig ist.

Satz. Gilt $D_{1}, D_{2} \in \mathscr{K}^{*}$ und $D, D^{\prime} \in \mathscr{U}_{*}\left(D_{1}, D_{2}\right)$, so ist $D \simeq D^{\prime}$ richtig.

Beweis. Es gelte $D_{0} \in \mathscr{l}_{*}\left(D, D^{\prime}\right)$. Wie in $\S 5$ angegeben, konstruiert man zu $D$ und $D^{\prime}$ unter Benutzung von $D_{0}$ eine minimale obere Schranke $D^{*}$. Bezeichnet $W$ die vermittelnde Abbildung der Beziehung $\left(L^{2}(X), U_{D}\right) \leqq\left(L^{2}\left(X^{*}\right), U_{D^{*}}\right)$ und $W^{\prime}$ die vermittelnde Abbildung für $\left(L^{2}\left(X^{\prime}\right), U_{D^{\prime}}\right) \leqq\left(L^{2}\left(X^{*}\right), U_{D^{*}}\right)$, so lassen sich folgende Eigenschaften zeigen:

1. Es gilt $H_{1}\left(D^{*}\right)=H_{1}(D)=H_{1}\left(D^{\prime}\right) \subseteq H_{1}\left(D_{i}\right)(i=1,2)$, wie man mit Hilfe des vorangegangenen Hilfssatzes beweist.

2. Es gilt $D^{*} \in \mathscr{K}^{*}$, wie aus der Torsionsfreiheit von $H_{1}\left(D^{*}\right)$, die durch die Eigenschaft 1 bewiesen wird, unmittelbar mit Hilfssatz 4 und 5 aus $\S 5$ folgt.

3. $\left\langle H_{2}\left(D^{*}\right), R_{D^{*}}\right\rangle \simeq\left\langle H_{2}(D), R_{D}\right\rangle \leqq\left\langle H_{2}\left(D_{i}\right), R_{D_{i}}\right\rangle(i=1,2)$ ist richtig. Das beweist man leicht mit Hilfe des vorangegangenen Hilfssatzes. Es gilt speziell $H_{2}\left(D^{*}\right)$. $=W H_{2}(D)$.

4. Für jedes $j \in \mathbf{N}$ gilt

$$
\left\langle H_{j}\left(D^{*}\right), R_{D^{*}}\right\rangle \simeq\left\langle\left\{W H_{j}(D), W^{\prime} H_{j}\left(D^{\prime}\right)\right\}, R_{D^{*}}\right\rangle .
$$

Ist nämlich

$$
W(h) \cdot W^{\prime}\left(h^{\prime}\right) \in H_{j}\left(D^{*}\right) . \quad\left(h \in H(D), h^{\prime} \in H\left(D^{\prime}\right)\right),
$$

richtig, so ist

$$
R_{D \cdot}^{j}\left(W(h) \cdot W^{\prime}\left(h^{\prime}\right)\right)=c \in H_{1}\left(D^{*}\right)=H_{1}(D)=H_{1}\left(D^{\prime}\right)
$$

gültig. Man erhält dạher $R_{D^{\bullet}}^{j}(W(h))=c \cdot R_{D^{\bullet}}^{j^{*}}\left(W^{\prime}\left(h^{\prime-1}\right)\right)$, und aus dem Vorangegangenen $R_{D^{*}} \mathbf{1}(W(h)) \in W H_{2}(D)$, d. h. $W(h) \in W H_{j}(D)$, und entsprechend $W^{\prime}\left(h^{\prime}\right) \in W^{\prime} H_{j}\left(D^{\prime}\right)$. Also gilt

$$
W(h) \cdot W^{\prime}\left(h^{\prime}\right) \in\left\{W H_{j}(D), W^{\prime} H_{j}\left(D^{\prime}\right)\right\}
$$

Umgekehrt ist offensichtlich auch

$$
\left\{W H_{j}(D), W^{\prime} H_{j}\left(D^{\prime}\right)\right\} \subseteq H_{j}\left(D^{*}\right)
$$

richtig. 
5. Für jedes $j \in \mathbf{N}$ ist $W H_{j}(D)=W^{\prime} H_{j}\left(D^{\prime}\right)$ gültig:

Die Behauptung sei für $j-1$ zutreffend. Ist $W(h) \cdot W^{\prime}\left(h^{\prime}\right) \in H_{j}\left(D^{*}\right)(h \in H(D)$, $h^{\prime} \in H\left(D^{\prime}\right)$ ) beliebig gewählt, so erhält man $W(h)=g \cdot W^{\prime}\left(h^{\prime}\right)$ mit $g \in W H_{j-1}(D)$ $=W^{\prime} H_{j-1}\left(D^{\prime}\right)$. (Vgl. Eigenschaft 4.) Das heißt, für jedes $W(h) \in W H_{j}(D)$ gilt

$$
W(h)=g \cdot W^{\prime}\left(h^{\prime}\right) \in\left\{W^{\prime} H_{j-1}\left(D^{\prime}\right), W^{\prime} H_{j}\left(D^{\prime}\right)\right\}=W^{\prime} H_{j}\left(D^{\prime}\right)
$$

und für jedes $W^{\prime}\left(h^{\prime}\right) \in W^{\prime} H_{j}\left(D^{\prime}\right)$

$$
W^{\prime}\left(h^{\prime}\right)=g^{-1} \cdot W(h) \in\left\{W H_{j-1}(D), W H_{j}(D)\right\}=W H_{j}(D) .
$$

Daraus folgt $W H_{j}(D)=W^{\prime} H_{j}\left(D^{\prime}\right)$.

Die Eigenschaften $1-5$ des Systems $D^{*}$ beweisen, daß $D \simeq D^{\prime}$ gelten muß.

\section{Obere Sehranken dynamischer Systeme aus $\mathscr{K}^{*}$}

Alternativ zur unteren Schranke dynamischer Systeme läßt sich zur Beschreibung der Menge aller dynamischen Systeme, die zwei oder mehr vorgegebene Systeme als Faktoren besitzen, der Begriff der oberen Schranke einführen. Er soll im folgenden für beliebige zwei Systeme aus $\mathscr{K}^{*}$ untersucht werden. Die angeführten Resultate lassen sich auch hier ohne Schwierigkeiten auf eine beliebige endliche Anzahl von Systemen aus $\mathscr{K}^{*}$ erweitern.

Definition. Sind $D_{1}, D_{2}$ Elemente aus $\mathscr{K}^{*}$, so wird ein dynamisches System $D_{0}$ abere Schranke von $D_{1}$ und $D_{2}$ genannt, wenn $D_{i} \leqq D_{0}(i=1,2)$ gilt. Ein dynamisches System $D_{0}$ wird minimale obere Schranke von $D_{1}$ und $D_{2}$ genannt, wenn gilt:

1. $D_{0}$ ist obere Schranke von $D_{1}$ und $D_{2}$.

2. Für jedes dynamische System $D$ mit der Eigenschaft $D_{i} \leqq D \leqq D_{0}(i=1,2)$ ist $D_{0} \simeq D$ richtig.

Bezeichnung. Dic Menge der oberen Schranken zweier Systeme $D_{1}$ und $D_{2}$ wird durch $\mathcal{O}\left(D_{1}, D_{2}\right)$ bezeichnet, die Menge der minimalen oberen Schranken durch, $\mathcal{O}^{*}\left(D_{1}, D_{2}\right)$.

Es ist offensichtlich, daß für beliebige Systeme $D_{1}, D_{2} \in \mathscr{K}^{*}$ stets $O\left(D_{1}, D_{2}\right) \neq \emptyset$ gilt, denn es ist z. B. immer $D_{1} \times D_{2} \in \mathcal{O}\left(D_{1}, D_{2}\right)$ richtig. Ebenso leicht überzeugt man sich, daß $\mathcal{O}\left(D_{1}, D_{2}\right)$ nicht in $\mathscr{K}^{*}$ enthalten ist: Bestehen die Mengen $X_{i}$ beide mod. Isomorphie aus genau einem Element, so ist jedes andere dynamische System, speziell also auch jedes nichtergodische System, obere Schranke von $D_{1}$ und $D_{2}$. Besitzt wenigstens eines der beiden Systeme $D_{i}$ eine nichttriviale Grundmenge $X_{i}$, o. B. d. A. sei es $D_{1}$, dann ist das System $D_{1} \times D_{1} \times D_{2}$ nichtergodische obere Schranke von $D_{1}$ und $D_{2}$.

Der nachfolgende Satz liefert ein Hilfsmittel zur Beschreibung der Menge $\mathcal{O}\left(D_{1}, D_{2}\right) \cap \mathscr{K}^{*}$.

Satz. Sind $D_{1}, D_{2}$ Elemente aus $\mathscr{K}^{*}$, so gilt $\mathcal{O}\left(D_{1}, D_{2}\right) \cap \mathscr{K}^{*} \neq \emptyset$ genau dann, wenn die erzeugte Gruppe $\left\{H_{1}\left(D_{1}\right), H_{1}\left(D_{2}\right)\right\}$ torsionsfrei ist.

Beweis.

1. Es wird vorqusgesetzt, daß die Gruppe $\left\{H_{1}\left(D_{1}\right), H_{1}\left(D_{2}\right)\right\}$ torsionsfrei ist. Man betrachtet das Produktsystem $D_{1} \times D_{2}$. Im Hilbertraum $L^{2}\left(X_{1} \times X_{2}\right)$ bilden dann die Funktionen $g_{1} \cdot g_{2}, g_{i} \in G\left(D_{i}\right) /_{k}(i=1,2)$ eine Orthonormalbasis. Die Elemente dieser Orthonormalbasis sind Quasieigenfunktionen in $L^{2}\left(X_{1} \times X_{2}\right)$ bezüglich des durch die Formel $U\left(g_{1} \cdot g_{2}\right)=U_{D_{2}}\left(g_{1}\right) \cdot U_{D_{1}}\left(g_{2}\right), g_{i} \in G\left(D_{i}\right)(i=1,2)$ 
definierten Automorphismus $U$. Die Abbildung $R_{1 \times 2}$, die durch die Beziehung

$$
R_{1 \times 2}\left(g_{1} \cdot g_{2}\right)=R_{D_{1}}\left(g_{1}\right) \cdot R_{D_{1}}\left(g_{2}\right), \quad g_{i} \in G\left(D_{i}\right) \quad(i=1,2)
$$

festgelegt ist, ist ein lokal-nilpotenter Endomorphismus auf der Gruppe $\left\{G\left(D_{1}\right), G\left(D_{2}\right)\right\}$ $\subseteq L^{2}\left(X_{1} \times X_{2}\right)$. Alle Elemente der erzeugten Gruppe

$$
\left\{H\left(D_{1}\right), H\left(D_{2}\right)\right\}=\left\{R_{D_{2}} G\left(D_{1}\right), R_{D_{2}} G\left(D_{2}\right)\right\}
$$

sind daher Quasieigenwerte in $L^{2}\left(X_{1} \times X_{2}\right)$ bezüglich $U$.

Offensichtlich ist $\left\{H\left(D_{1}\right), H\left(D_{2}\right)\right\}$ kommutativ und abzählbar. Bezüglich des Endomorphismus $R_{1 \times 2}$ ist diese Gruppe torsionsfrei :

Zunächst zeigt sich, daß der Kern von $R_{1 \times 2}$ bezüglich $\left\{H\left(D_{1}\right), H\left(D_{2}\right)\right\}$ enthalten ist in der erzeugten Gruppe $\left\{H_{2}\left(D_{1}\right), H_{2}\left(D_{2}\right)\right\}$. Gilt nämlich

d. h.

$$
R_{1 \times 2}\left(h_{1} \cdot h_{2}\right)=1 \text {, }
$$

$$
R_{1 \times 2}\left(h_{1}\right)=R_{D_{1}}\left(h_{1}\right)=R_{D_{2}}\left(h_{2}^{-1}\right)=R_{1 \times 2}\left(h_{2}^{-1}\right),
$$

$h_{i} \in H\left(D_{i}\right)(i=1,2)$, so ist für die eindeutigen Darstellungen

und

$$
R_{1 \times 2}\left(h_{1}\right)=c_{1} \cdot h_{1} \cdot 1
$$

$$
R_{1 \times 2}\left(h_{2}^{-1}\right)=c_{2} \cdot 1 \cdot f_{2}, \quad c_{i} \in K, \quad f_{i} \in G\left(D_{i}\right) /_{K} \quad(i=1,2),
$$

$c_{1} \cdot f_{1} \cdot 1=c_{2}: 1 \cdot f_{2}$ richtig. Wegen der Eindeutigkeit der Darstellung durch Elemente der Orthonormalbasis für jede Funktion aus $L^{2}\left(X_{1} \times X_{2}\right)$ ergibt sich $f_{1}=1$ und $f_{2}=1$. Es gilt daher $R_{D_{1}}\left(h_{1}\right)=c_{1}$, d. h. $h_{1} \in H_{2}\left(D_{1}\right)$, und $R_{D_{2}}\left(h_{2}\right)=c_{2}^{-1}$, d. h. $h_{2} \in H_{2}\left(D_{2}\right)$.

Die erzeugte Gruppe $\left\{H_{2}\left(D_{1}\right), H_{2}\left(D_{2}\right)\right\}$ ist torsionsfrei. Ist nämlich $h_{1} \cdot h_{2}$ $\epsilon\left\{H_{2}\left(D_{1}\right), H_{2}\left(D_{2}\right)\right\}$ ein Gruppenelement mit der Eigenschaft

$$
\left(h_{1}: h_{2}\right)^{n}=1 \text { für gewisses } n \in \mathbf{Z} \backslash\{0\},
$$

so zeigt man unter Benutzung der angegebenen Orthonormalbasis $h_{1}{ }^{n}=h_{2}{ }^{-n}$ $=c \in K$. Wegen der Torsionsfreiheit der Gruppen $H\left(D_{i}\right)(i=1,2)$ gilt dann $R_{D_{1}}\left(h_{1}\right)=1$ und $R_{D_{1}}\left(h_{2}^{-1}\right)=1$, d. h. $h_{1} \in H_{1}\left(D_{1}\right)$ und $h_{2}^{-1} \in H_{1}\left(D_{2}\right)$. Die vorausgesetzte Torsionsfreiheit der Gruppe $\left\{H_{1}\left(D_{1}\right), H_{1}\left(D_{2}\right)\right\}$ liefert daher für alle Funktionen $h_{1} \cdot h_{2}, h_{i} \in H_{2}\left(D_{i}\right)(i=1,2)$, für die ein $n \in Z \backslash\{0\}$ mit $\left(h_{1} \cdot h_{2}\right)^{n}=1$ existiert, folgende notwendige Beziehung: $h_{1} \cdot h_{2}=1$. Mithin ist $\left\{H_{2}\left(D_{1}\right), H_{2}\left(D_{2}\right)\right\}$ torsionsfrei.

Die Torsionsfreiheit der Gruppe $\left\{H\left(D_{1}\right), H\left(D_{2}\right)\right\}$ folgt aus der Torsionsfreiheit der Gruppe $\left\{H_{2}\left(D_{1}\right), H_{2}\left(D_{2}\right)\right\}$ : Ist $h \in\left\{H\left(D_{1}\right), H\left(D_{2}\right)\right\}$ eine Funktion mit der Eigenschaft $h^{n}=1$ für gewisses $n \in \mathbf{Z} \backslash\{0\}$, so läßt sich, falls $R_{1 \times 2}(h) \neq 1$ gilt, wegen der lokalen Nilpotenz von $R_{1 \times 2}$ eine natürliche Zahl $m$ so bestimmen, daß

$$
R_{1 \times 2}^{m+1}(h)=1 \quad \text { und } \quad R_{1 \times 2}^{m}(h)=f \neq 1, \quad f \in\left\{H_{2}\left(D_{1}\right), H_{2}\left(D_{2}\right)\right\} \text { ， }
$$

richtig ist. Die Voraussetzung über $h$ führt zu dem Widerspruch

$$
1=R_{1 \times 2}^{m}(1)=R_{1 \times 2}^{m}\left(h^{n}\right)=\left(R_{1 \times 2}^{m}(h)\right)^{n}=f^{n} \neq 1 .
$$

Gilt andernfalls unter der Voraussetzung $h^{n}=1, n \neq 0$, die Beziehung $R_{1 \times 2}(h)=1$, so folgt $h=1$, wie bereits oben gezeigt wurde.

Man betrachtet nun das Paar $\left\langle\left\{H\left(D_{1}\right), H\left(D_{2}\right)\right\}, R_{1 \times 2}\right\rangle$. Falls der Kern von $R_{1 \times 2}$ in $\left\{H\left(D_{1}\right), H\left(D_{2}\right)\right\}$ keine Untergruppe der Gruppe $K$ ist, so bildet man $\left\{H\left(D_{1}\right), H\left(D_{2}\right)\right\}$ vermittels eines Isomorphismus $\varphi$, $\operatorname{der} \operatorname{der}$ Beziehung $\varphi(c)=c$ für alle Elemente $c$ 
aus $\left\{H_{1}\left(D_{1}\right), H_{1}\left(D_{2}\right)\right\}$ genügt, in $K$ ab. Die Existenz eines solchen Isomorphismus $\varphi$ ist wegen der Eigenschaften der Gruppe $\left\{H\left(D_{1}\right), H\left(D_{2}\right)\right\}$ gesichert. (Vgl. [1], S. 519, Lemma 3.)

Durch die Bedingung

$$
R(f)=R(\varphi(h))=\varphi\left(R_{1 \times 2}(h)\right), \quad h \in\left\{H\left(D_{1}\right), H\left(D_{2}\right)\right\}, \quad f=\varphi(h),
$$

wird ein lokal-nilpotenter Endomorphismus $R$ auf $\varphi\left(\left\{H\left(D_{1}\right), H\left(D_{2}\right)\right\}\right)$ definiert. Aus den oben bewiesenen Eigenschaften folgt, daß $\left\langle\left\{H\left(D_{1}\right), H\left(D_{2}\right)\right\}, R_{1 \times 2}\right\rangle$ (bzw. $\left\langle\varphi\left(\left\{H\left(D_{1}\right), H\left(D_{2}\right)\right\}\right), R\right\rangle$, falls eine Transformation erforderlich sein sollte) eine Endomorphismus-Gruppe bildet. Jedes dynamische System $D \in \mathscr{K}^{*}$, für das die Beziehung

$$
\left\langle H(D), R_{D}\right\rangle \simeq\left\langle\left\{H\left(D_{1}\right), H\left(D_{2}\right)\right\}, R_{1 \times 2}\right\rangle
$$

(bzw. $\left.\left\langle H(D), R_{D}\right\rangle \simeq\left\langle\varphi\left(\left\{H\left(D_{1}\right), H\left(D_{2}\right)\right\}\right), R\right\rangle\right)$ richtig ist, ist obere Schranke von $D_{1}$ und $D_{2}$.

2. Es wird vorausgesetzt, daß die Gruppe $\left\{H_{1}\left(D_{1}\right), H_{1}\left(D_{2}\right)\right\}$ nicht torsionsfrei ist. Existierte ein System $D \in \mathscr{K}^{*}$ nit der Eigenschaft $D_{i} \leqq D(i=1,2)$, so müßten die Beziehungen $\left\langle H\left(D_{i}\right), R_{D_{1}}\right\rangle \leqq\left\langle H(D), R_{D}\right\rangle$, mit den vermittelnden Abbildungen $V_{i}: H\left(D_{i}\right) \rightarrow H(D) \quad(i=1,2)$ richtig sein. Es müßte also insbesondere $H_{1}\left(D_{i}\right)$ $=V_{i} H_{1}\left(D_{i}\right) \subseteq H_{1}(D)(i=1,2)$, d. h. $\left\{H_{1}\left(D_{1}\right), H_{1}\left(D_{2}\right)\right\} \subseteq H_{1}(D)$, gelten. Die Voraussetzung steht dann aber im Widerspruch zur Torsionsfreiheit der Gruppe $H_{1}(D)$.

Bemerkung. Man überzeugt sich leicht, daß es dynamische Systeme $D_{1}, D_{2} \in \mathscr{K}^{*}$ mit der Eigenschaft $\mathcal{O}\left(D_{1}, D_{2}\right) \cap \mathscr{K}^{*}=\emptyset$ gibt. Man betrachte z. B. zu beliebigem System $D_{1} \in \mathscr{K}^{*}$ ein $D_{2} \in \mathscr{K}^{*}$, das die folgende Eigenschaft besitzt: Es gibt eine Konstante $c_{1} \in H_{1}\left(D_{1}\right)$ so, daß $c_{2}=c \cdot c_{1}$ für ein Element $c_{2} \in H_{1}\left(D_{2}\right)$ gilt, wobei $c$ eine $n$-te Einheitswurzel aus $K$ ist $(c \neq 1, n \neq 0)$. Die Gruppe $\left\{H_{1}\left(D_{1}\right), H_{1}\left(D_{2}\right)\right\}$ enthält dann mit $c$ ein Element endlicher Ordnung, ist also nicht torsionsfrei.

Für die Menge der oberen Schranken zweier Systeme $D_{1}, D_{2} \in \mathscr{K}^{*}$ war die Eigenschaft $\mathcal{O}\left(D_{1}, D_{2}\right) \neq \varnothing$ offensichtlich. Der Nachweis der Richtigkeit der Bedingung $\mathcal{O}^{*}\left(D_{1}, D_{2}\right) \neq \varnothing$ soll konstruktiv erfolgen. In den Beweis wird ein Element $D_{0}$ der Menge $\mathscr{U}_{*}\left(D_{1}, D_{2}\right)$ einbezogen. Nach einem Satz von H. FurstenberG ([4], S. 5) existiert eine obere Schranke $D$ von $D_{1}$ und $D_{2}$ derart, daß $\left(L^{2}\left(X_{0}\right), U_{D_{0}}\right) \leqq\left(L^{2}\left(X_{i}\right), U_{D_{i}}\right)$ mit der vermittelnden Abbildung $V_{i}: L^{2}\left(X_{0}\right) \rightarrow L^{2}\left(X_{i}\right)$ und $\left(L^{2}\left(X_{i}\right), U_{D_{1}}\right)$ $\leqq\left(L^{2}(X), U_{D}\right)$ mit der vermittelnden Abbildung $W_{i}: L^{2}\left(X_{i}\right) \rightarrow L^{2}(X) \quad(i=1,2)$, mit der Bedingung $W_{1} V_{1}\left(f_{0}\right)=W_{2} V_{2}\left(f_{0}\right)$ für alle Funktionen $f_{0} \in L^{2}\left(X_{0}\right)$ richtig ist. Man betrachtet den unitären Unterring $\mathscr{H}$ von $L^{2}(X)$, der durch die erzeugte Gruppe $\left\{W_{1} G\left(D_{1}\right), W_{2} G\left(D_{2}\right)\right\}$ aufgespannt wird. Das mod. Isomorphie eindeutig bestimmte dynamische System, dessen Spektraltyp zu $\left(\mathscr{H}, U_{D}\right)$ äquivalent ist, soll durch das Symbol $D^{\prime}$ bezeichnet werden. Die folgenden Hilfssätze zeigen einige Eigenschaften von $\left(\mathscr{H}, U_{D}\right)$. (Es werden dabei die angegebenen Bezeichnungen und Zusammenhänge benutzt.)

Hilfssatz 1. Es gilt $W_{1} H\left(D_{1}\right) \cap W_{2} H\left(D_{2}\right)=W_{1} V_{1} H\left(D_{0}\right)=W_{2} V_{2} H\left(D_{0}\right)$.

Hilfssat z 2. Gilt für zwei Funktionen $g_{1} \in G\left(D_{1}\right), g_{2} \in G\left(D_{2}\right)$ die Beziehung $R_{D}\left(W_{1} g_{1}\right)$ $=R_{D}\left(W_{2} g_{2}\right)$, so existiert ein Element $g_{0} \in G\left(D_{0}\right)$ derart, da $\beta g_{1}=V_{1}\left(g_{0}\right)$ und $g_{2}=c \cdot V_{2}\left(g_{0}\right)$, $c \in \mid K$, richtig ist.

Hilfssatz 3. Die Funktionen $\left.f_{1} \cdot f_{2} \in\left\{W_{1} G\left(D_{1}\right), W_{2} G\left(D_{2}\right)\right\}\right\}_{K}, \quad f_{i} \in W_{i} G\left(D_{i}\right)$ $(i=1,2)$ bilden in $\mathscr{H}$ ein Orthonormalsystem.

Durch $R_{D}\left(f_{1} \cdot f_{2}\right)=W_{1} R_{D_{1}}\left(g_{1}\right) \cdot W_{2} R_{D_{3}}\left(g_{2}\right), f_{i}=W_{i}\left(g_{i}\right), g_{i} \in G\left(D_{i}\right)(i=1,2)$ wird auf der Gruppe $\left\{W_{1} G\left(D_{1}\right), W_{2} G\left(D_{2}\right)\right\}$ ein Endomorphismus bestimmt, der jeder Funktion dieser Gruppe ihren Quasieigenwert bezüglich $U_{D}$ zuordnet. 
Hilfssatz 4. Für jeden Quasieigenwert $h \in\left\{W_{1} G\left(D_{1}\right), W_{2} G\left(D_{2}\right)\right\}$ in $\mathscr{H}$ existiert eine Funktion $g \in\left\{W_{1} G\left(D_{1}\right), W_{2} G\left(D_{2}\right)\right\} /_{K}$ mit der Eigenschaft $R_{D}(g)=h$.

Hilfssatz 5. Der unitäre Ring $\mathscr{H}$ mit dem aufgeprägten Automorphismus $U_{D}$ ist ergodisch.

Für beliebige zwei Systeme $D_{1}, D_{2} \in \mathscr{K}^{*}$ existiert also stets eine ergodische obere Schranke mit quasidiskretem Spektrum. Der folgende Satz benutzt das System $D^{\prime}$, um die Beziehung $\mathcal{O}^{*}\left(D_{1}, D_{2}\right) \neq \varnothing$ nachzuweisen.

Satz. Für beliebige zwei Systeme $D_{1}, D_{2} \in \mathscr{K}^{*}$ gilt $\mathcal{O}^{*}\left(D_{1}, D_{2}\right) \neq \varnothing$.

Beweis. Man konstruiert, wie oben angegeben, zu $D_{1}$ und $D_{2}$ das System $D^{\prime}$. Dann gilt $D^{\prime} \in \mathcal{O}^{*}\left(D_{1}, D_{2}\right): D^{\prime} \in \mathcal{O}\left(D_{1}, D_{2}\right)$ ist aus der Konstruktion offensichtlich. Ist $D^{\prime \prime} \in \mathcal{O}\left(D_{1}, D_{2}\right)$ ein dynamisches System mit der Eigenschaft $D^{\prime \prime} \leqq D^{\prime}$, so folgt $D^{\prime \prime} \simeq D^{\prime}$, wie man auf nachfolgende Weise einsieht.

Es gelte

$\left(L_{2}\left(X_{i}\right), U_{D_{i}}\right) \leqq\left(L^{2}\left(X^{\prime \prime}\right), U_{D^{\prime \prime}}\right)$ mit der vermittelnden Abbildung

$Z_{i}: L^{2}\left(X_{i}\right) \rightarrow L^{2}\left(X^{\prime \prime}\right)$,

$\left(L^{2}\left(X^{\prime \prime}\right), U_{D^{\prime \prime}}\right) \leqq\left(\mathscr{H}, U_{D}\right) \simeq\left(L^{2}\left(X^{\prime}\right), U_{D^{\prime}}\right)$ mit der vermittelnden Abbildung $Z: L^{2}\left(X^{\prime \prime}\right) \rightarrow \mathscr{H}$,

$\left(L^{2}\left(X_{0}\right), U_{D_{0}}\right) \leqq\left(L^{2}\left(X_{i}\right), U_{D_{1}}\right)$ mit der vermittelnden Abbildung

$V_{i}: L^{2}\left(X_{0}\right) \rightarrow L^{2}\left(X_{i}\right)$ und

$\left(L^{2}\left(X_{i}\right), U_{D_{i}}\right) \leqq\left(\mathscr{H}, U_{D}\right)$ mit der vermittelnden Abbildung

$W_{i}: L^{2}\left(X_{i}\right) \rightarrow \mathscr{H} \quad(i=1,2)$.

1. Sind $g_{i} \in G\left(D_{i}\right) /_{K}(i=1,2)$ zwei Funktionen mit der Eigenschaft $W_{1}\left(g_{1}\right)$ $=W_{2}\left(g_{2}\right)$, so gilt auch $Z Z_{1}\left(g_{1}\right)=Z Z_{2}\left(g_{2}\right)$. Sind nämlich die Funktionen $g_{i}$ Elemente von $G_{1}\left(D_{i}\right) /_{K}(i=1,2)$, so folgt aus der Ergodizität von $U_{D}$, und den Hilfssätzen 1-3 leicht die Behauptung. Durch Induktion nach der Ordnung der Quasieigenfunktionen zeigt man, ebenfalls unter Heranziehung der Ergodizität von $U_{D}$, und der Hilfssätze 1-3, die Richtigkeit der Aussage für beliebige Quasieigenfunktionen $g_{i} \in G\left(D_{i}\right) /_{\kappa}$ mit der Eigenschaft $W_{1}\left(g_{1}\right)=W_{2}\left(g_{2}\right)$.

2. Die Gruppe $G\left(D_{0}\right) /_{K}$ kann vermittels der multiplikativen, isometrischen Operatoren $Z Z_{1} V_{1}$ und $Z Z_{2} V_{2}$ in die Gruppe $\left\{Z Z_{1} G\left(D_{1}\right) /_{K}, Z Z_{2} G\left(D_{2}\right) /_{K}\right\}$ isomorph eingebettet werden. Beweisteil 1 zeigt, daß $Z Z_{1} V_{1}\left(g_{0}\right)=Z Z_{2} V_{2}\left(g_{0}\right)$ für alle Funktionen $g_{0} \in G\left(D_{0}\right) /_{K}$ richtig ist.

3. Nimmt man an, daß $D^{\prime \prime}<D^{\prime}$ gilt, so ergeben sich folgende Zusammenhänge: Aus Hilfssatz 4 und 5 folgt, da $\beta$ die Gruppe $\left\{W_{1} G\left(D_{1}\right), W_{2} G\left(D_{2}\right)\right\}$ alle Quasieigenfunktionen aus $\mathscr{H}$ enthält. Da die Abbildung $Z \cdot$ die Faktorbeziehung zwischen $\left(L^{2}\left(X^{\prime \prime}\right), U_{D^{\prime \prime}}\right)$ und $\left(\mathscr{H}, U_{D}\right)$ vermittelt, besitzt sie speziell auch die Eigenschaft, die Gruppe der Quasieigenfunktionen $\left\{Z_{1} G\left(D_{1}\right), Z_{2} G\left(D_{2}\right)\right\}$ in die Gruppe $\left\{W_{1} G\left(D_{1}\right)\right.$, $\left.W_{2} G\left(D_{2}\right)\right\}$ abzubilden. Wegen der Annahme muß dann

$$
\left\{Z Z_{1} G\left(D_{1}\right), Z Z_{2} G\left(D_{2}\right)\right\} \subset\left\{W_{1} G\left(D_{1}\right), W_{2} G\left(D_{2}\right)\right\}
$$

richtig sein. Daher läßt sich die Gruppe $\left\{Z Z_{1} G\left(D_{1}\right) /_{K}, Z Z_{2} G\left(D_{2}\right) /_{K}\right\}$ durch einen injektiven Homomorphismus stets nur in die Gruppe $\left\{W_{1} G\left(D_{1}\right) /_{K}, W_{2} G\left(D_{2}\right) /_{K}\right\}$ abbilden. Es existieren daher Funktionen $g_{i} \in G\left(D_{i}\right) / K(i=1,2)$ mit den Eigenschaften

$$
\begin{aligned}
& W_{1}\left(g_{1}\right) \neq W_{2}\left(g_{2}\right), \\
& Z Z_{1}\left(g_{1}\right)=Z Z_{2}\left(g_{2}\right) .
\end{aligned}
$$


Es seien $g_{1}, g_{2}$ so ausgewählt, daß sie den Beziehungen (1) und (2) genügen. Für die erzeugte Gruppe $\left\{Z Z_{1} V_{1} G\left(D_{0}\right) /_{K}, Z Z_{1}\left(g_{1}\right)\right\}$ gilt dann:

$$
\begin{aligned}
& H\left(D_{0}\right) \simeq Z Z_{1} V_{1} G\left(D_{0}\right) /_{K} \subset\left\{Z Z_{1} V_{1} G\left(D_{0}\right) /_{K}, Z Z_{1}\left(g_{1}\right)\right\}, \\
& \left.\left\{Z Z_{1} \nabla_{1} G\left(D_{0}\right), Z Z_{1}\left(g_{1}\right)\right\}\right|_{K} \simeq\left\{Z Z_{1} V_{1} H\left(D_{0}\right), Z Z_{1} R_{D_{1}}\left(g_{1}\right)\right\},
\end{aligned}
$$

(vgl. [1], S. 516), und

$$
\left\{Z Z_{1} V_{1} H\left(D_{0}\right), Z Z_{1} R_{D_{1}}\left(g_{1}\right)\right\}=\left\{Z Z_{2} V_{2} H\left(D_{0}\right), Z Z_{2} R_{D_{2}}\left(g_{2}\right)\right\} \text {. }
$$

Daher sind die folgenden Beziehungen richtig:

$$
\left\langle Z Z_{1} V_{1} H\left(D_{0}\right), R_{D^{\prime}}\right\rangle<\left\langle\left\{Z Z_{1} V_{1} H\left(D_{0}\right), Z Z_{1} R_{D_{1}}\left(g_{1}\right)\right\}, R_{D^{\prime}}\right\rangle \leqq\left\langle Z Z_{1} H\left(D_{1}\right), R_{D^{\prime}}\right\rangle
$$

und

$$
\begin{aligned}
\left\langle\left\{Z Z_{1} V_{1} H\left(D_{0}\right), Z Z_{1} R_{D_{1}}\left(g_{1}\right)\right\}, R_{D^{\prime}}\right\rangle & \simeq\left\langle\left\{Z Z_{2} V_{2} H\left(D_{0}\right), Z Z_{2} R_{D^{\prime}}\left(g_{2}\right)\right\}, R_{D^{\prime}}\right\rangle \\
& \leqq\left\langle Z Z_{2} H\left(D_{2}\right), R_{D}\right\rangle .
\end{aligned}
$$

Es existiert also ein System $D_{0}^{\prime} \in \mathscr{K}^{*}$, das folgende Eigenschaften hat:

$$
\begin{aligned}
\left\langle H\left(D_{0}\right), R_{D_{0}}\right\rangle\left\langle\left\langle H\left(D_{0}{ }^{\prime}\right), R_{D^{\prime}}\right\rangle\right. & \simeq\left\langle\left\langle Z Z_{1} V_{1} H\left(D_{0}\right), Z Z_{1} R_{D_{1}}\left(g_{1}\right)\right\}, R_{D^{\prime}}\right\rangle \\
& \leqq\left\langle H\left(D_{i}\right), R_{D_{i}}\right\rangle \quad(i=1,2) .
\end{aligned}
$$

Das widerspricht aber der Voraussetzung $D_{0} \in \mathscr{U}_{*}\left(D_{1}, D_{2}\right)$. Es gilt folglich

$$
\left\{Z Z_{1} G\left(D_{1}\right), Z Z_{2} G\left(D_{2}\right)\right\}=\left\{W_{1} G\left(D_{1}\right), W_{2} G\left(D_{2}\right)\right\} \text {. }
$$

Der von der Gruppe $\left\{Z Z_{1} G\left(D_{1}\right), Z Z_{2} G\left(D_{2}\right)\right\}$ erzeugte unitäre Ring $\mathscr{H}_{1}$ stimmt daher mit $\mathscr{H}$ uberein. Damit ergibt sich $\mathscr{H}=\mathscr{H}_{1} \subseteq Z L^{2}\left(X^{\prime \prime}\right) \subseteq \mathscr{H} . D^{\prime \prime}$ und $D^{\prime}$ sind also isomorphe dynamische Systeme.

Satz. Gilt für zwei Systeme $D_{1}, D_{2}$ aus $\mathscr{K}^{*}$ die Beziehung $\mathcal{O}\left(D_{1}, D_{2}\right) \cap \mathscr{K}^{*} \neq \varnothing$, so ist auch $\mathcal{O}^{*}\left(D_{1}, D_{2}\right) \cap \mathscr{K}^{*} \neq \varnothing$ richtig.

Beweis. Die Menge $\mathscr{H}=\mathscr{O}\left(D_{1}, D_{2}\right) \cap \mathscr{K}^{*}$, ausgestattet mit der Faktorbeziehung als Ordnungsrelation, ist eine induktiv geordnete Menge. Ist nämlich $\left(D_{i}{ }^{\prime}\right)_{i \in I}$ eine beliebige (fallend geordnete) Kette aus $\mathscr{A}$, gilt also

$$
\left\langle H\left(D_{i+1}^{\prime}\right), R_{D_{i+1}^{\prime}}\right\rangle \leqq\left\langle H\left(D_{i}^{\prime}\right), R_{D_{i}}\right\rangle
$$

mit der vermittelnden Abbildung $V_{i}: H\left(D_{i+1}^{\prime}\right) \rightarrow H\left(D_{i}{ }^{\prime}\right)(i \in I)$, so ist jedes dynamische System $D$, dessen Endomorphismus-Gruppe äquivalent ist zu

$$
\left\langle\bigcap_{\substack{i \in I \\ i \neq 1}}\left(\prod_{j=1}^{i-1} V_{j}\right) H\left(D_{i}{ }^{\prime}\right) \cap H\left(D_{1}{ }^{\prime}\right), R_{D_{1}^{\prime}}\right)
$$

untere Grenze der betrachteten Kette in $\mathscr{M}$. (Dabei bezeichne $\prod_{j=1}^{i-1} V_{j}$ die Hintereinanderausführung der Homomorphismen $V_{j}(j=1, \ldots, i-1)$.) Die Anwendung des Zornschen Lemmas ergibt, daß in $\mathscr{M}$ ein bezüglich der betrachteten Ordnungsrelation minimales Element $D_{0}$ existiert. Da die Klasse $\mathscr{K}^{*}$ faktorerblich ist, ist $D_{0}$ zugleich auch minimal in $\mathcal{O}\left(D_{1}, D_{2}\right)$. Es gilt also $D_{0} \in \mathcal{O}^{*}\left(D_{1}, D_{2}\right) \cap \mathscr{K}^{*}$.

Satz. Gilt $D_{1}, D_{2} \in \mathscr{K}^{*}$, so enthält die Menge $\mathcal{O}^{*}\left(D_{1}, D_{2}\right) \cap \mathscr{K}^{*}$, sofern sie nicht leer ist, mod. Isomorphie genau ein Element.

Beweis. Es gelte $D, D^{\prime} \in \mathcal{O}^{*}\left(D_{1}, D_{2}^{\prime}\right) \cap \mathscr{X}^{*}$. Für jedes System $\tilde{D} \in \mathscr{U}_{*}\left(D, D^{\prime}\right)$ trifft dann, da $D_{1}, D_{2} \in \mathscr{U}\left(D, D^{\prime}\right)$ richtig ist, $D_{i} \leqq \widetilde{D}(i=1,2)$ zu. Wegen $D, D^{\prime}$ $\in \mathcal{O}^{*}\left(D_{1}, D_{2}\right)$ folgt daraus sofort $\tilde{D} \simeq D^{\prime}$ und $\tilde{D} \simeq \bar{D}$, d. h. $D \simeq D^{\prime}$. 
Bemerkung. Die von W. Parry in der Arbeit [11] durchgeführten Untersuchungen für spezielle obere Schranken unipotenter affiner Transformationen lassen. sich in die vorliegenden Betrachtungen auf folgende Weise einordnen:

Man prüft leicht nach, daß für zwei Systeme $D_{1}, D_{2} \in \mathscr{K}^{*}$ genau dann eine vollergodische obere Schranke existiert, wenn $\mathcal{O}\left(D_{1}, D_{2}\right) \cap \mathscr{K}^{*} \neq \varnothing$ gilt. (Sei nämlich $\mathcal{O}\left(D_{1}, D_{2}\right) \cap \mathscr{K}^{*}=\emptyset$, d. h. $\left\{H_{1}\left(D_{1}\right), H_{1}\left(D_{2}\right)\right\}$ eine nichttorsionsfreie Gruppe, dann enthält der Hilbertraum $L^{2}(X)$ jeder vollergodischen oberen Schranke $D$ von $D_{1}$ und $D_{2}$ einen Eigenwert endlicher Ordnung (bezüglich $U_{D}$ ): Ist $c=c_{1} \cdot c_{2}$ $=R_{D_{1}}\left(f_{1}\right) R_{D_{2}}\left(f_{2}\right)$ eine $n$-te Einheitswurzel, $(n \neq 0)$, dann gilt

$$
\begin{aligned}
& U_{D}\left(S_{1} f_{1} \cdot S_{2} f_{2}\right)=c \cdot\left(S_{1} f_{1}\right) \cdot\left(S_{2} f_{2}\right) \quad\left(c_{i} \in H_{1}\left(D_{i}\right), f_{i} \in G\left(D_{i}\right),\right. \\
& R_{D_{1}}\left(f_{i}\right)=c_{i} \quad(i=1,2),
\end{aligned}
$$

wenn $S_{i}$ die Faktorabbildungen zwischen $D_{i}$ und $D$ darstellen. Die Existenz eines Eigenwertes endlicher Ordnung widerspricht aber der vorausgesetzten Vollergodizität von $D$. Die Richtigkeit der umgekehrten Behauptung ist offensichtlich.)

Benutzt man die Bezeichnung

$\mathscr{A}=$ Menge aller dynamischen Systeme, die eine Darstellung durch eine unipotente affine Transformation auf einer Nilmannigfaltigkeit besitzen, so liefert Theorem 2.2 aus [11] für Elemente aus $\mathscr{K}^{*}$ folgendes Resultat:

Satz. Für beliebige zwei Systeme $D_{1}, D_{2} \in \mathscr{K}^{*} \cap \mathscr{H}$ mit $\mathcal{O}\left(D_{1}, D_{2}\right) \cap \mathscr{K}^{*} \neq \varnothing$ existiert stets ein minimales Element in $\mathcal{O}\left(D_{1}, D_{2}\right) \cap \mathscr{H}$.

(Minimalität ist hier bezüglich der Faktorrelation gemeint.)

Unter den im Satz angegebenen Voraussetzungen für $D_{1}, D_{2} \in \mathscr{K}^{*}$ ergibt sich daruber hinaus mit Hilfe des zitierten Theorems von W. Parry, daß jedes vollergodische System aus $\mathcal{O}^{*}\left(D_{1}, D_{2}\right)$ eine Darstellung als unipotente affine Transformation auf einer Nilmannigfaltigkeit besitzt. Das Ergebnis von W. ParRy ermöglicht also, für spezielle Systeme aus $\mathscr{K}^{*}$ Eigenschaften der Menge ihrer minimalen oberen Schranken von einem andersgearteten Aspekt aus, als es in der vorliegenden Arbeit getan wird, zu beschreiben.

\section{LITERATUR}

[1] Аврамов, Л. М.: Метрические автоморфизмы с.квазидискретным спектром. Изв. AH CCCP 26 (1962), 513-530.

[2] Brown, J. R.: Inverse limits, entropy and weak isomorphism for discrete dynamical systems. Transactions Amer. Math. Soc. 164 (1972), 55-66.

[3] Drnculeanu, N., and C. Foras: Algebraic models for measure preserving transformations. Michigan Math. J. 15 (1968), 215-237.

[4] Furstenbero, H.: Disjointness in ergodic theory, minimal sets and a problem in diophantine approximation. Math. Systems Theory 1 (1967), 1-49.

[5] HARN, F., and W. PARRY: Some characteristic properties of dynamical systems with quasidiscrete spectrum. Math. Systems Theory 2 (1968), 179-190.

[6] Хеслер, К.: Дизьюнктные вполне әргодические динамические системы с квазидискретпым спектром. Вестник ЛГУ 19 (1980).

[7] JACOBs, K.: Lecture Notes in Ergodic Theory 1962/63, Part II. 1. Aufl., Aarhus 1963.

[8] J J COBS, K.: Recent Developments in Ergodic Theory. In: Transactions of the Seventh Prague Conference on Information Theory, Statistical Decision Functions, Random Processes 1974, Vol. A. Prague 1977, p. 283-308.

[9] Ornstein, D.: Bernoulli shifts with the same entropy are isomorphic. Adv. in Math. 4 (1970), 337-352. 
[10] Ornstein, D.: Ergodic theory, randomness and dynamical systems. New Haven and London 1974.

[11] PArry, W.: Dynamical representations in nilmanifolds. Comp. mat. 26 (1973), 159-174.

[12] Пинскер, М. С.: Димамические системы с вполне положительноп и нулевой энтропией. Докл. АН СССР 133 (1960), 1025-1026.

[13] Синай, Я. Г.: Слабып изозорфивм преобравований с инвариантнои мерой. Докл. AH CCCP 147 (1962), 797-800.

Manuskripteingang: 31. 7. 1980

\section{VERFASSER :}

Dr. KARIN HäsLer

Sektion Mathematik der Martin-Luther-Universität Halle-Wittenberg DDR - 4010 Halle, Universitätsplatz 6 